\title{
An improved immunofluorescent reagent for rapid, direct detection of Bordetella pertussis
}

\author{
Denis Martin Phd, Patricia McNicol PhD, Richard Marchand md, Pierre Lebel Md, \\ MARK S PEPPLER D PHIL, BERNARD R BRODEUR PHD
}

I NFECTION WITH BORDETELLA PERTUSSIS IS STILL AN IMPORTANT public health problem, and outbreaks of pertussis continue to occur in Canada. In 1992, over 3700 cases of pertussis infection were reported in Canada (1). Moreover, 7049 and 3218 cases of pertussis were reported for 1993 and the first half of 1994, respectively, indicating a rise in the incidence of this disease in Canada (personal communication). The incidence of this disease varies by age group, but is highest in infants less than a year old, with an annual rate reaching one case per 1000. The incidence of pertussis in adolescents and adults is underreported, in part because symptoms are often less characteristic than with childhood disease. Yet adolescents and adults act as an important reservoir for transmitting disease to susceptible populations of young children $(2,3)$. Recently, the first case of $B$ pertussis disease caused by a strain highly resistant to erythromycin, the antibiotic of choice against pertussis, was reported in the United States (4). It is estimated that approximately $0.04 \%$ of infected children in developed countries die every year from this disease and its consequences, especially pneumonia (1). In general,

National Laboratory for Immunology, Laboratory Centre for Disease Control, Ottawa, Ontario; Cadham Provincial Laboratory, Winnipeg, Manitoba; Département de Microbiologie et Immunologie, Hôpital Ste-Justine, Montréal, Québec; and Department of Medical Microbiology and Infectious Diseases, University of Alberta, Edmonton, Alberta

Correspondence and reprints: Dr BR Brodeur, Chief, National Laboratory for Immunology, Laboratory Centre for Disease Control, HPB Bldg 7, Tunney's Pasture, Ottawa, Ontario K1A OL2 the currently used whole-cell pertussis vaccines are efficacious as indicated by a reduction of $93 \%$ in annual incidence compared with the prevaccine period. However, pertussis is still a major concern and was ranked as the fifth priority disease for national surveillance by the Canadian Advisory Committee on Epidemiology. In 1993, a consensus conference on pertussis was sponsored by the Childhood Immunization Division, Bureau of Communicable Disease Epidemiology, Laboratory Centre for Disease Control (LCDC) to establish national goals and recommendations for the control of pertussis. One of the recommendations specifically stated that the development and standardization of rapid diagnostic tests were urgently required.

Isolation of $B$ pertussis from nasopharyngeal swabs or aspirations remains the 'gold standard' for the diagnosis of pertussis infection (5). This method is highly specific but is time-consuming, labour intensive and results are obtained only after four to seven days of culture on specialized media. Culture is available in many clinical laboratories but has a low sensitivity of 40 to $60 \%$ (5). Serological analysis by a variety of methods is available, but the requirement for seroconversion generally delays the detection of pertussis-specific antibodies beyond the time by which diagnosis is required $(2,6)$. These serological assays are better suited for epidemiological studies of pertussis infections. Polymerase chain reaction (PCR)-based identification systems are under development and evaluation, but are not readily available (7). These assays will likely become essential tools for the diagnosis of pertussis in the future and will probably have a detection capability beyond that observed for culture.

Direct immunofluorescent assay with polyvalent serum is 
used to obtain an early presumptive diagnosis or to confirm culture. However, cross-reactions of the polyvalent serum with other bacterial species or other antigens present in the clinical specimen have resulted in a very high incidence of false positive results, with rates as high as $85 \%$ depending on the expertise of the clinical laboratory personnel (6). One way to improve significantly the performance of direct immunofluorescent assay is to develop highly specific immunological reagents, such as high affinity monoclonal antibodies (MAbs). Already, MAb-based diagnostic tests are being used with great success in clinical laboratories.

Identification of the most suitable target antigen(s) for a particular diagnostic test is a key step in the successful production of highly specific MAbs. The lipo-oligosaccharide (LOS) of $B$ pertussis is a desirable antigen for such a purpose because it is one of the essential components of the outer membrane and is therefore present at the surface of all organisms isolated from active infection. A series of MAbs, named $\mathrm{BL}-1$ to $\mathrm{BL}-7$, which recognize the Los of $B$ pertussis and do not cross-react with other nasopharyngeal bacteria, was developed by the National Laboratory for Immunology, LCDC (8). Subsequently, one of these MAbs was used to generate a specific fluorescein-labelled immunological reagent for direct immunofluorescent assay.

The unique antigenic site recognized by the MAbs is located on three amino sugars of the carbohydrate portion of $B$ pertussis LOS (9). This reactive site is antigenically very stable and highly conserved among all $B$ pertussis isolates regardless of physiological state. Only very rare laboratory Los variant strains do not have these three amino sugars on the LOS molecule and are therefore not recognized by the MAbs. A radioimmunobinding assay was used to demonstrate that this antigenic site is freely accessible at the surface of intact bacteria and could be detected directly in clinical specimens without the need to disrupt partially cell integrity. Two subsequent experiments, in vitro bactericidal assay and protection in animal aerosol model, further demonstrated the importance and the surface accessibility of this antigenic site $(10,11)$.

To develop a direct immunofluorescent assay, one of these MAbs was purified and subsequently coupled to fluorescein-isothiocyanate. The fluorescein-labelled MAb was first tested against a panel of $B$ pertussis strains obtained after culture on Bordet-Gengou agar, as well as other bacterial species normally found in the nasopharynx. It was observed that a blocking step with $10 \%$ rabbit serum eliminated the nonspecific binding of the MAb Fc region to receptors found on certain bacterial cells. The labelled MAb specifically recognized all $64 B$ pertussis clinical isolates without any cross-reaction with Bordetella parapertussis or any other bacterial species normally isolated from the respiratory tract. Weakly fluorescent bacterial cells were observed when the labelled MAb was incubated with Bordetella bronchiseptica strains. This reactivity was not surprising since antigenic relationships between the Los of $B$ pertussis and the lipopolysaccharide of $B$ bronchiseptica have been previously reported (9). $B$ bronchiseptica is an animal pathogen that rarely causes infection in humans exposed to infected animals (12).
The Cadham Provincial Laboratory in Manitoba in collaboration with the National Laboratory for Immunology are evaluating the sensitivity and specificity of this immunological reagent directly with clinical specimens obtained during an outbreak of pertussis. The study is designed to compare culture with the direct detection of $B$ pertussis on smears obtained from nasopharyngeal swabs by the fluorescein-labelled MAb. Already, 1398 smears have been tested and preliminary results indicate that the sensitivity of the direct immunofluorescent assay compared with culture is $65 \%$, while the specificity recorded is as high as $99.6 \%$. This latter result suggests that the use of a more refined immunological reagent can significantly increase the specificity of direct immunofluorescent assay compared with the results obtained with fluorescein-labelled polyvalent antisera. It is important to note that only five of the 1398 specimens tested gave positive results while the culture remained negative. To verify whether $B$ pertussis cells are present or absent on these slides, each sample is eluted and then tested by PCR.

Recently, it was observed that patients for whom diagnosis of infectious diseases was made more rapidly had a significantly lower mortality rate (13). Furthermore, rapid diagnosis of $B$ pertussis helps not only to improve treatment, but also to initiate chemoprophylaxis rapidly to close contacts of the patient (14). For these reasons, we are also developing an ultrarapid automated assay using flow cytometry in collaboration with the Department of Microbiology and Immunology of the Ste-Justine Hospital in Montreal. There are two major advantages to an automated assay over traditional direct immunofluorescent assay. First is a significant increase in accuracy because the readings would be standardized, reproducible and not depend on the competence of a specialized technologist. Second would be a noticeable reduction in the time normally required to process and analyze a specimen.

Flow cytometry can be easily adapted for the accurate detection of $B$ pertussis cells directly in clinical specimens. Flow cytometers are designed to count particles efficiently by size and protein content using light scattering. In our case, the instrument was adjusted by preliminary experiments to detect particles matching $B$ pertussis cell size and nucleic acid content. To increase the specificity of the test, the fluoresceinlabelled MAb is then used to tag the $B$ pertussis organisms. Thus, only fluorescent $B$ pertussis cells are counted. All other particles are ignored.

To date, 125 clinical samples obtained from patients with clinical signs compatible with pertussis infection have been processed and analyzed using a flow cytometer. These analyses facilitated optimizing the procedure to obtain the best results with a minimum number of steps. It was noted that the bacteria present in nasopharyngeal swabs could be directly suspended in phosphate-buffered saline. On the contrary, the mucoid substances always present in the nasopharyngeal aspirations had to be liquified with dithiothreitol and then filtered before they could be labelled by the MAb. The flow cytometer was always calibrated with standardized positive and negative controls to obtain comparable readings from 
one run to another. Preliminary results indicate that this ultrarapid automated fluorescent assay (less than 45 mins from beginning to end) is at least as sensitive as culture. Moreover, several culture-negative samples were clearly categorized as positive by this assay, suggesting that this assay may be more sensitive than culture. PCR amplification of $B$ pertussis DNA will again be used to confirm the presence of $B$ pertussis cells not detected by culture. These preliminary experiments

\section{RERERECES}

1. Pertussis Consensus Conference. Can Dis Wkly Rep 1994;19:124-35.

2. Hodder SL, Mortimer EA Jr. Epidem iology of pertus sis and reactions to pertussis vaccine. Epidemiol Rev 1992;14:243-67.

3. Cherry JD. Pertussis: the trials and tribulations of old and new pertussis vaccines. Vaccine 1992;10:1033-8.

4. Erythromycin-resistant $B$ pertus s is - Yuma County, Arizona, May-October 1994. MMWR 1994;43/44:807-10.

5. Gilchrist MJR. Bordetella. In: Balows A, Hausler WJ Jr, Herrmann KL, Is en berg HD, Shadomy HJ, eds. Manual of Clinical Microbiology, 5 th edn. Wash ington: American Society for Microbiology, 1991:471-7.

6. Ewanowich CA, Chui LW-L, Paranchych MG, Peppler MS, Marusyk RG, Albritton WL. Major outbreak of pertuss is in northern Alberta, Canada: Analysis of discrepant direct fluorescent-antibody and culture results by using polymerase chain reaction methodology. J Clin Microbiol 1993;31:1715-25.

7. Meade BD, Bollen A. Recommendations for use of the polymerase chain reaction in the diagn os is of Bordetella pertuss is infections. J Med Microbiol 1994;41:51-5. clearly suggest that it is possible to use this automated assay to identify $B$ pertussis rapidly - within an hour - giving the clinician additional criteria to establish an early diagnosis. This automated process may be very useful for the rapid screening of large numbers of samples during major outbreaks.

8. Archambault D, Rondeau P, Martin D, Brodeur BR Characterization and comparative bactericidal activity of monoclonal antibodies to Bordetella pertus sis lipo-oligosaccharide A. J Gen Microbiol 1991;137:905-11.

9. Martin D, Peppler MS, Brodeur BR. Immunological characterization of the lipooligosaccharide B band of Bordetella pertussis. In fect Immun 1992;60:2718-25.

10. Brodeur BR, Hamel J, Martin D, Rondeau P. Biological activity of a human monoclonal antibody to Bordetella pertuss is lipooligosaccharide. Hum Antibodies Hybridomas 1991;2:194-9.

11. Shah in RD, Hamel J, Leef MF, Brodeur BR. Analys is of protective and non protective monoclonal antibodies specific for Bordetella pertuss is lipooligosaccharide. In fect Immun 1994;62:722-5.

12. Goodnow RA. Biology of Bordetella bronchis eptica. Microbiol Rev 1980;44:722-38.

13. Doern GV, Vautour R, Gaudet M, Levy B. Clin ical impact of rapid in vitro susceptibility testing and bacterial identification. J Clin Microbiol 1994;32:1757-62.

14. Spawer MA, Cochi SL, Zell ER, et al. Prevention of secondary transmission of pertuss is in households with early use of erythromycin. Am J Dis Child 1992;146:177-81. 


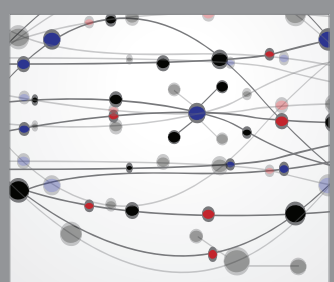

The Scientific World Journal
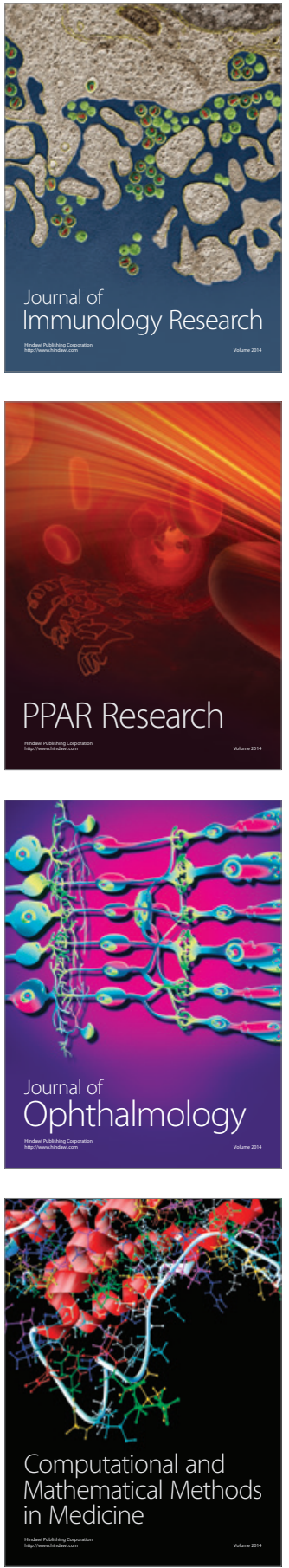

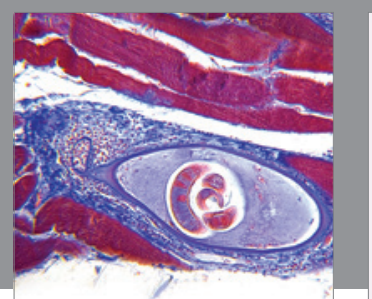

Gastroenterology Research and Practice

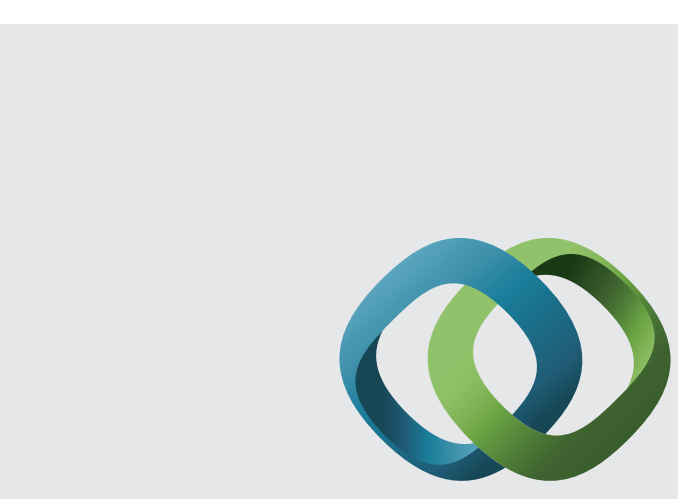

\section{Hindawi}

Submit your manuscripts at

http://www.hindawi.com
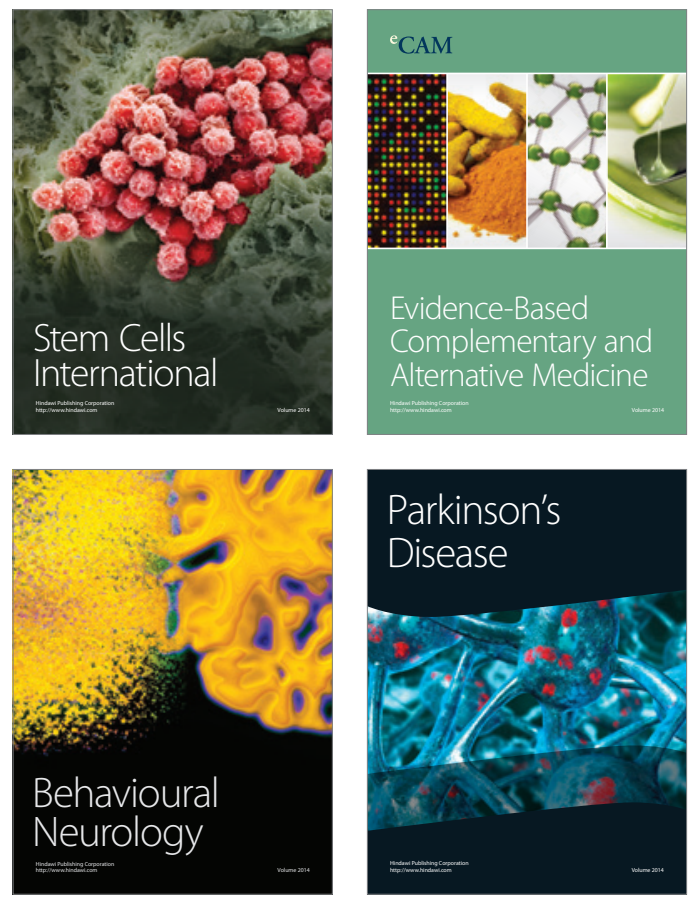
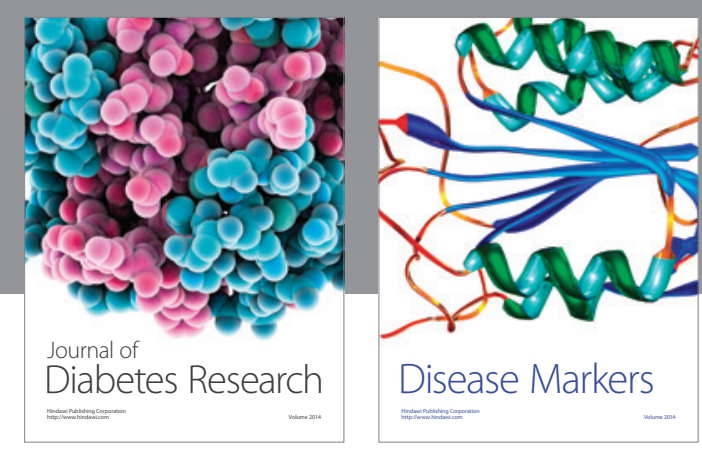

Disease Markers
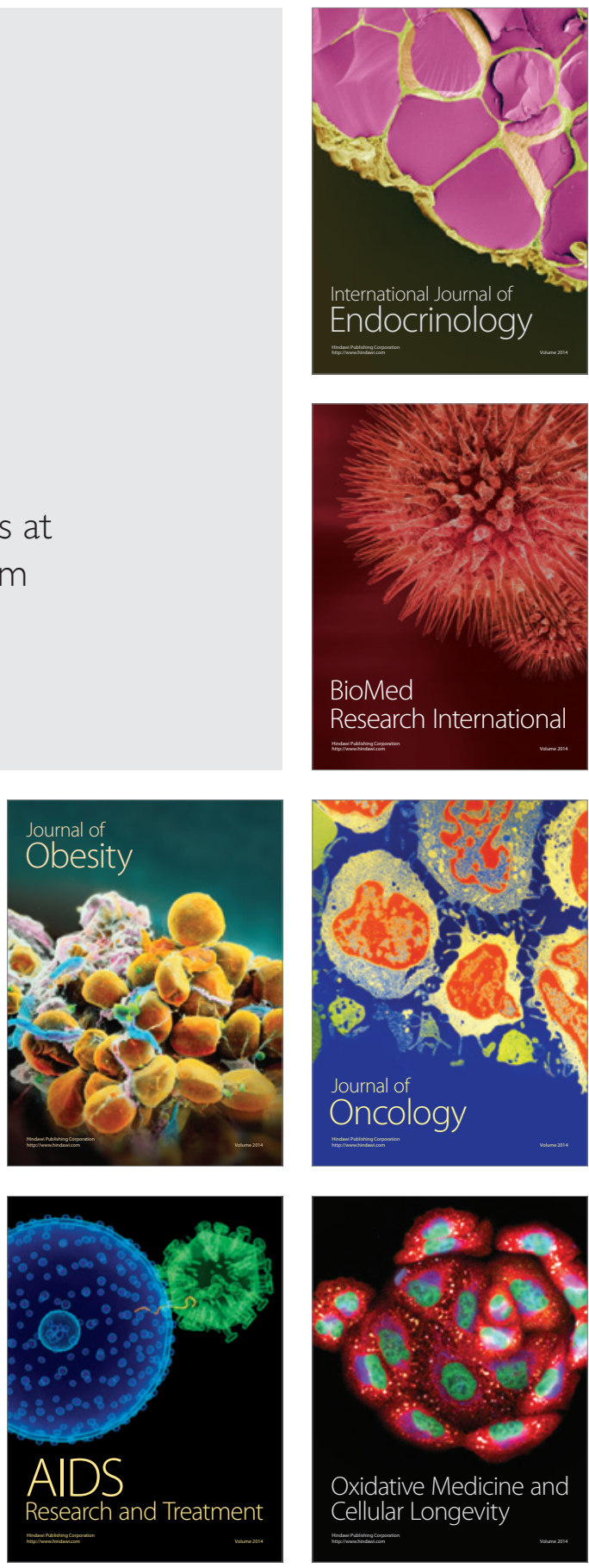